\title{
Strong coupling between surface plasmon-polaritons and organic molecules in subwavelength hole arrays
}

\author{
J. Dintinger, ${ }^{1}$ S. Klein,,$* *$ F. Bustos,${ }^{1, \dagger}$ W. L. Barnes, ${ }^{2}$ and T. W. Ebbesen ${ }^{1, \ddagger}$ \\ ${ }^{1}$ ISIS, Université Louis Pasteur, 8 allée G. Monge, 67000 Strasbourg, France \\ ${ }^{2}$ School of Physics, University of Exeter, Stocker Road, Exeter EX4 4QL, United Kingdom \\ (Received 4 August 2004; revised manuscript received 11 November 2004; published 28 January 2005)
}

\begin{abstract}
The interaction of a $J$-aggregate and surface plasmon polariton modes of a subwavelength hole array have been studied in detail. By measuring the effects of hole array period, angular dispersion and concentration of the $J$-aggregate on the transmission of the array, the existence of a strong coupling regime is demonstrated with a Rabi splitting of $250 \mathrm{meV}$. This large splitting is explained not only by the high oscillator strength of the dye but also by the high local field amplitudes generated by surface plasmons of the metallic structure.
\end{abstract}

DOI: 10.1103/PhysRevB.71.035424 PACS number(s): 73.20.Mf, 71.36.+c, 71.35.Cc, 78.66.Qn

\section{INTRODUCTION}

The ability to texture metals down to the nanoscale has opened new perspectives in the field of photonics. ${ }^{1}$ In order to create new miniaturized devices, surface plasmon-based photonics or plasmonics take advantage of the particular behaviour of the bound waves that exist at the surface of nanostructured metals such as surface plasmon-polaritons (SPPs). Metallic films periodically milled with subwavelength holes are an example of such a device where coupling of light to SPPs enhances the optical transmission by orders of magnitude (see, for example, Refs. 2-6). Beyond their fundamental interest, subwavelength hole arrays are increasingly being used for spectroscopic and sensor applications (see, for example, Refs. 7-10). In such cases, it is important to understand the details of the interaction between the molecules and the SPPs sustained by the hole arrays. Both the properties of molecules and those of the SPPs can be strongly affected by each other. For instance, molecular fluorescence is modified by proximity to a metal surface ${ }^{11-13}$ and the surface plasmonpolariton dispersion curves of flat metallic films are changed by the presence of molecular absorption transitions. ${ }^{14,15}$

For those reasons, we have studied in detail the interaction of dye molecules with the SPPs of subwavelength hole arrays. In the process we have found that, under certain conditions, a strong coupling regime between the dye and SPP modes can be observed as reported in this article. Strong coupling has been the subject of much research in atomic and solid state physics ${ }^{16-20}$ and has been studied more recently with molecular excitons. ${ }^{21-23}$ The coupling between the photonic mode and the electronic transition in these systems is such that new mixed states, termed cavity polaritons, are formed. The system response is then to oscillate in time between the two states (empty cavity with excited dye and cavity photon with relaxed dye states), illustrating the periodical exchange of photon energy between the coupled modes. The period of these so-called Rabi oscillations have to be shorter than the lifetime of the two states for the system to be in the oscillatory regime. Typical evidence of the strong coupling regime (in the static domain) is the anticrossing behavior in the dispersion curve of the photon mode at the exciton energy where otherwise the uncoupled modes would have crossed.
In the present case, the role of the cavity or waveguide mode is played by the hole array SPP modes and the exciton is a $J$-aggregate that has proven to be well suited for strong coupling studies. ${ }^{22}$

\section{SAMPLE FABRICATION}

The samples used in this work were prepared as follows. A quartz substrate was coated with a $270 \mathrm{~nm}$ thick Ag film after which the hole array was milled with a focused ion beam (FEI Dual Strata 235); see Fig. 1. A range of samples were made with different hole periods between $290 \mathrm{~nm}$ and $450 \mathrm{~nm}$, the hole period to diameter ratio being kept at 2.5 whilst the period was varied. A $300 \mathrm{~nm}$ thick polyvinyl alcohol (PVA) film, both with and without the $J$-aggregate (2,2'-dimethyl-8-phenyl-5,6,5', 6' -dibenzothiacarbocyanine chloride from Hayashibara Biochemical Laboratories, Inc.) was then spin-coated on the samples. The typical concentration of the $J$-aggregate in the dry PVA was 0.5 moles per $\mathrm{dm}^{3}$.

\section{RESULTS AND DISCUSSION}

Subwavelength hole arrays in optically thick metal films, as shown in Fig. 1, are characterized by transmission peaks with transmittivity far greater than expected for such small apertures. The enhanced transmission is due to the coupling

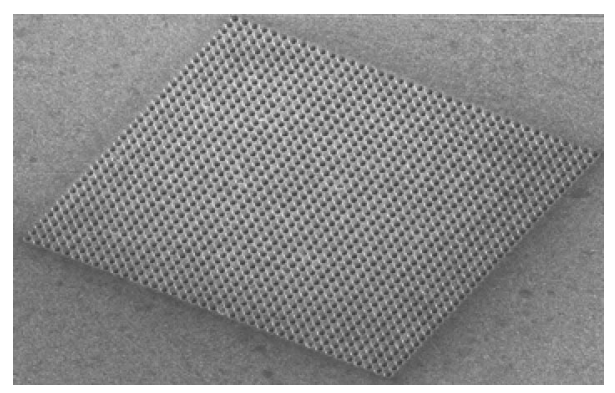

FIG. 1. Scanning electron image of a typical hole array milled in a silver film (thickness $=370 \mathrm{~nm}$; period $=380 \mathrm{~nm}$; diameter $=150 \mathrm{~nm}$ ). 


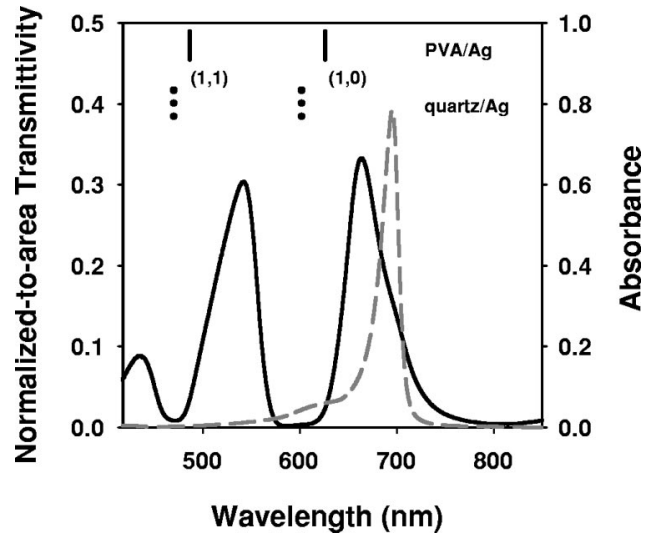

FIG. 2. Normal incidence transmission spectrum of a silver hole array (period $=380 \mathrm{~nm}$ ) covered with a 300 -nm-thick PVA film (black), and absorption spectrum of a PVA film doped with the cyanine dyes (dashed gray line). Markers indicate the wavelengths of the different SPP modes estimated from Eq. (1) and identified by the indices $(i, j)$.

of light to surface plasmon-polariton modes resulting in large field amplitudes at the surface which compensate for the otherwise inefficient tunneling process through the apertures. This event occurs whenever the energy and in-plane momentum of light and the SPP mode are identical. SPPs possess more momentum than freely propagating light, thus the matching conditions are provided by the Bragg scattering induced by the periodicity of the structure as described by the following equation: ${ }^{3}$

$$
\left|\overrightarrow{k_{S P}}\right|=\left|\overrightarrow{k_{\|}}+i \overrightarrow{G_{x}}+j \overrightarrow{G_{y}}\right|,
$$

where $k_{S P}$ is the surface plasmon-polariton wave vector, $k_{\|}$is the inplane wave vector component of the incident light, $G_{x}$ and $G_{y}$ are the reciprocal lattice vectors $\left(\left|G_{x}\right|=\left|G_{y}\right|=2 \pi / P, P\right.$ being the period of the array), and $i, j$ are integers.

For a square array at normal incidence, it can be shown that the peak positions are then given in a first approximation by the following equation:

$$
\lambda_{\max } \sqrt{\left(i^{2}+j^{2}\right)}=P \sqrt{\frac{\varepsilon_{1} \cdot \varepsilon_{2}}{\varepsilon_{1}+\varepsilon_{2}}},
$$

where $\varepsilon_{1}$ and $\varepsilon_{2}$ are the dielectric constant of the metal and the dielectric media, respectively. Each set of indices $(i, j)$ is therefore associated with a peak in the transmission spectrum as shown in Fig. 2. Note that SPP modes appear at both metal/dielectric interfaces of the film and that these are normally offset due to the difference in refractive index of the two dielectric media. The small refractive index contrast between the quartz substrate (1.46) and the PVA (ca. 1.52) coating used here results in the appearance of a single set of peaks.

The $J$-aggregate is characterized by a strong and sharp absorption peak at $693 \mathrm{~nm}(1.78 \mathrm{eV})$ also shown in Fig. 2. When it is present at high concentrations in the PVA film on top of the hole array, the transmission spectrum of the latter at normal incidence changes dramatically, as illustrated in Fig. 3. As can be seen, the original peak is split into two

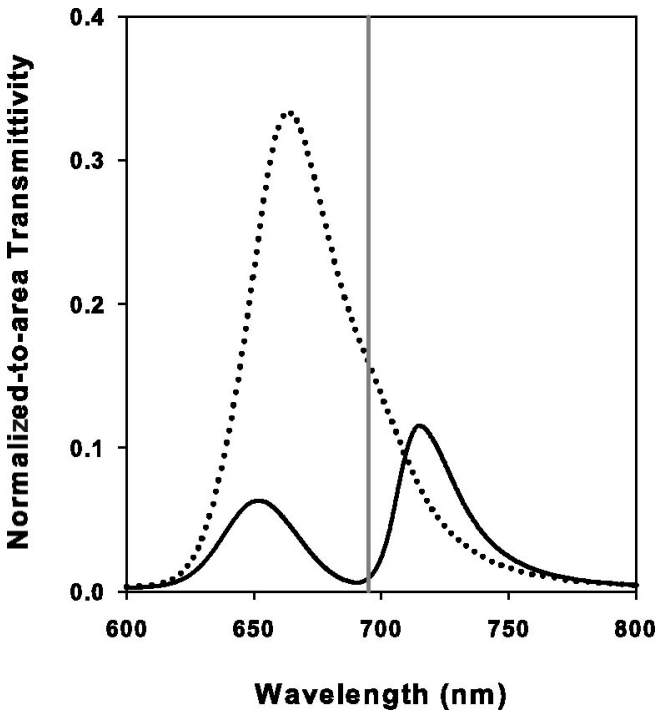

FIG. 3. Normal incidence transmission spectra of a hole array $(P=380 \mathrm{~nm})$ covered with undoped PVA (dotted line) and with $J$-aggregate doped PVA (solid line). The vertical line at $693 \mathrm{~nm}$ indicates the peak of the $J$-aggregate absorption band.

peaks whose positions depend on the SPP mode energy relative to that of the exciton. We must emphasize here that this gap does not result simply from the damping of the transmitted light by absorption due to the molecules; similar results are observed whether or not absorption is taken into account in plotting the transmission spectra. (For clarity the data presented here were not corrected.)

To fully analyze the interaction between the molecules and the SPP modes, it is instructive to vary in a systematic way the energy of the SPP mode relative to the dispersionless exciton band. In this way, we can probe the response of the system when the two modes are brought into resonance. By virtue of Eq. (1), the resonance and therefore the strong coupling can be studied by either varying the period of the hole array or the angle of incidence of the incoming light on the sample. These two methods provide complementary evidence of the strong coupling regime, as shown next.

\section{A. Dependence of normal incidence transmission spectra on hole period}

Figure 3 shows the splitting of the $(1,0)$ SPP peak in the presence of the $J$-aggregate. In Fig. 4 the peak positions are plotted for a wide range of array periods (between $290 \mathrm{~nm}$ and $450 \mathrm{~nm}$ ), and show the typical signature of strong coupling: anticrossing. As can be seen, the original dispersion curve of the $(1,0)$ SPP mode (in white) undergoes anticrossing around the exciton energy. The resonance, characterized by a minimum splitting energy difference, occurs for a period of $380 \mathrm{~nm}$. This is slightly lower than the $400 \mathrm{~nm}$ indicated by the undoped samples spectra, and is probably due to the presence of the $(1,0)$ mode at the quartz interface; this mode induces a slight blue shift of the apparent $(1,0)$ peak compared to a truly symmetric structure (two PVA/silver interfaces). In other words, only the $(1,0)$ mode on PVA side is affected by the close proximity of the $J$-aggregate. It should 


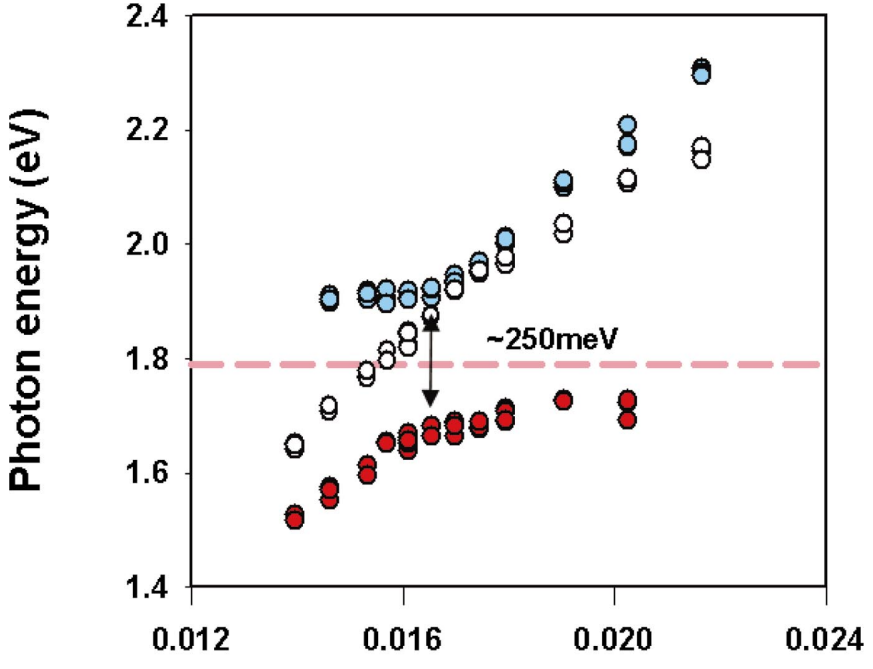

\section{In-plane momentum $\left(\mathrm{nm}^{-1}\right)$}

FIG. 4. (Color) Energy dispersion curves for undoped samples (white) and $J$-aggregate doped samples (red and blue) determined by varying the period of the hole arrays. The blue and red dots correspond to the two peaks shown in Fig. 3 with an extended range of period (from 290 to $450 \mathrm{~nm}$ ), and the red dashed line to the exciton absorption energy.

also be noted that equal or similar linewidths and intensities of the two peaks are not observed exactly on resonance $(P$ $=380 \mathrm{~nm}$ ) but for a slightly positive energy detuning, as a consequence of the asymmetric inhomogeously broadened $J$-aggregate absorption. ${ }^{24,25}$

The corresponding Rabi splitting measured on resonance (roughly $250 \mathrm{meV}$ ) is in good agreement with the results obtained with similar dyes in Fabry-Pérot microcavities. ${ }^{22,23}$

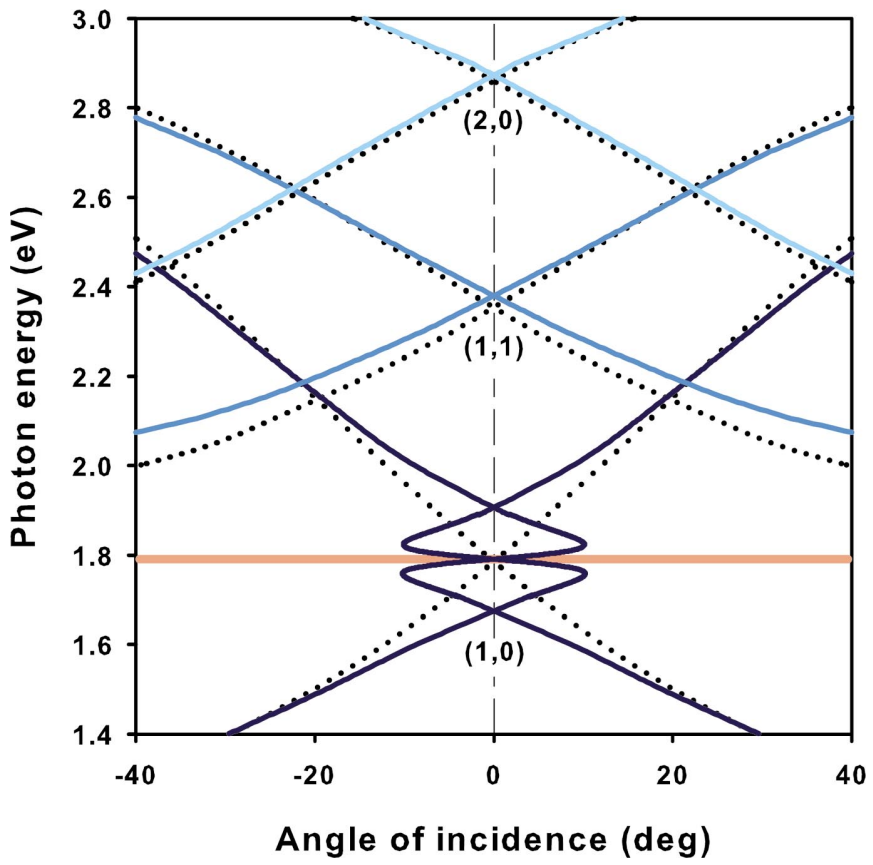

FIG. 5. (Color) SPP dispersion curves, for a silver/PVA (black dotted) and silver/J-aggregate doped PVA interface (blue curves). These plots were done using Eqs. (1) and (2); the dielectric constant of silver was taken from Ref. 29 and that of the dye was estimated from a simple Drude-Lorentz model using its known absorption properties. The period $P$, used for these calculations, was chosen such that the $(1,0)$ modes of the PVA/silver interface cross the dispersionless exciton band (red line) at normal incidence.

The calculated lifetime of the hybrid polariton from this width ( $\sim 3 \mathrm{fs})$ is indeed shorter than the lifetime of the uncoupled states (on the order of 10 fs for $\mathrm{SPP}^{26,27}$ and on the order of ps for $J$-aggregates ${ }^{28}$ ).

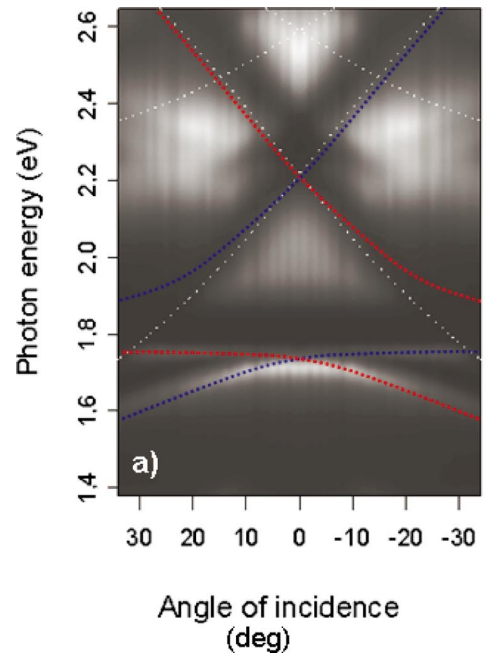

(deg)

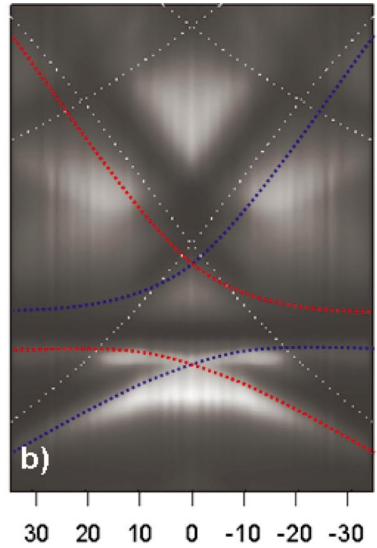

Angle of incidence (deg)

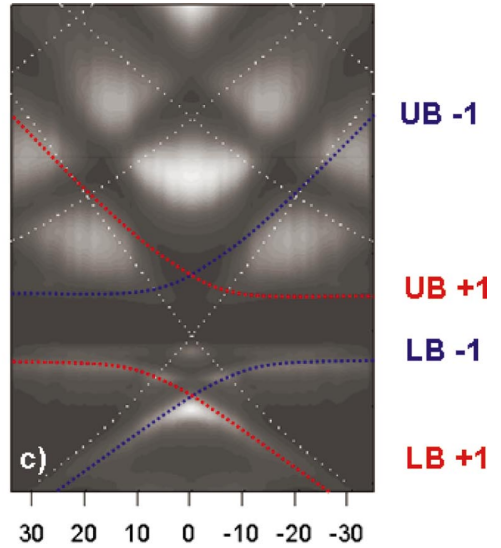

Angle of incidence (deg)

FIG. 6. (Color) Measured transmittance of $p$-polarized light as a function of angle of incidence and photon energy for different hole arrays with period 330 (a), 380 (b), and 430 (c) nm. The data are presented using a grey-intensity scale (white stands for high transmission while black for low transmission). White dotted lines represent the theoretical dispersion curves of the SPP mode propagating at the PVA/silver interface in the absence of dye; the blue and red curves are only guides for the eye to show the two upper and lower polariton branches (UB and LB). Note that the data were mainly collected for positive angles and the symmetrical representation is only used for clarity. 


\section{B. Angle dependence of transmission spectra}

Using Eq. (1), one can calculate the SPP dispersion curves of the hole arrays in the presence of the exciton but in the absence of strong coupling. For instance, Fig. 5 shows the results of such a calculation where the exciton is at resonance with the $(1,0)$ SPP mode at normal incidence. The wiggle at the exciton energy is due to the dispersion of the dielectric constant induced around the exciton absorption, something we estimated using the Drude-Lorentz model. When the dye concentration is sufficiently high in the PVA film on the hole array, the experimentally derived dispersion curves are very different from such predictions and reveal the expected anticrossing due to strong coupling as can be seen in Fig. 6. Data are shown for 3 different array periods chosen to be negatively detuned, positively detuned and on resonance relative to the exciton energy at normal incidence. Superimposed on these experimental data are the calculated dispersion curves of the SPP modes on the PVA side of the hole array (for clarity in the absence of the $J$-aggregate). The colored dashed lines are schematic dispersion curves as a guide to the eyes of the changes induced by the strong coupling.

All the experimental dispersion curves shown in Fig. 6 show the opening of a gap at the exciton energy and the resulting band splitting and bending. The two lower polariton branches ( $\mathrm{LB}+1$ and $\mathrm{LB}-1)$, originating from the modes $(+1,0)$ and $(-1,0)$, are clearly observed below the $J$-aggregate band which is centered around $1.78 \mathrm{eV}$. In the transmission spectra they appear as a couple of separated diverging peaks whose progression toward high energies is blocked by the exciton band. As a consequence the lower branches are progressively flattened as the period is reduced owing to this band being closer to the exciton energy, as can be seen by comparing the curvature of the LBs with decreasing period in Fig. 6. In the case of the two upper polariton branches ( $\mathrm{UB}+1$ and $\mathrm{UB}-1$ ), the situation is complicated by the nearby presence of other modes such as the $\operatorname{SPP}(1,1)$ modes and in-plane interference phenomena of the various SPP modes (which is beyond the scope of this article). The splitting between upper and lower polariton branches, on resonance, is again ca. $250 \mathrm{meV}$ as expected from the results of Fig. 4.

\section{Dependence of the splitting on concentration}

As a final evidence of strong coupling, we measured the dependence of the Rabi splitting energy with the exciton density by varying the $J$-aggregate concentrations in the PVA

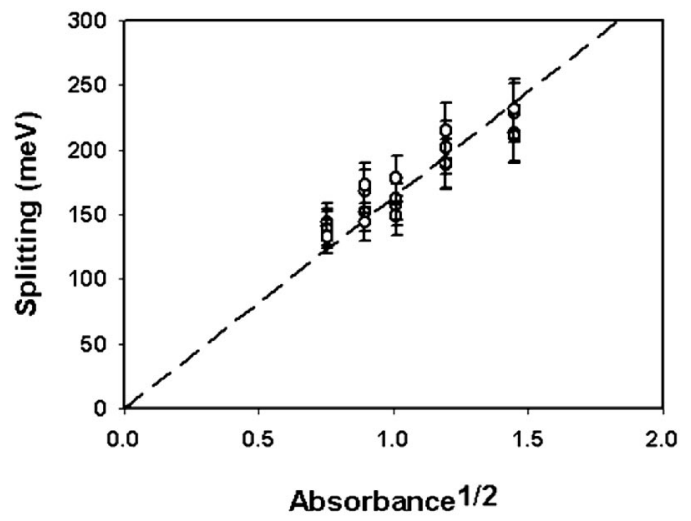

FIG. 7. Variation of the Rabi splitting as a function of the square root of the absorbance of the $J$-aggregate/PVA film.

films. The splitting energy is expected to vary as the square root of the absorbance, which itself is the product of the film thickness, the concentration and the intrinsic absorption cross-section of the $J$-aggregate. The results plotted in Fig. 7 show very good agreement with the predicted square root dependence which has also been been observed in the case of dyes in microcavities. ${ }^{21,23}$

The above evidence indicates that dye molecules such as $J$-aggregates can undergo strong coupling with the surface plasmon-polariton modes supported by the subwavelength hole arrays. At appropriate frequencies these modes are standing waves on the surface of the array and may thus act in a similar fashion to the cavity in strong coupling experiments involving microcavities. Despite their low $Q$ factor (ca. 10), strong coupling is still observed because of the very large oscillator strength of the dye and the fact that the SPPs are associated with enhanced electric fields in the immediate vicinity of the surface where the dye is located. ${ }^{23}$ This combination also results in the large Rabi splitting $(250 \mathrm{meV})$. In general, surface plasmon-polaritons which can confine the electromagnetic energy in very small volumes, should be very favorable to strong coupling. ${ }^{30}$ It is possible that periodic metal gratings also allow the observation of strong coupling. Therefore, the possibility of such interactions must be considered when studying molecule-surface plasmonpolariton phenomena in nanostructured systems.

\section{ACKNOWLEDGMENTS}

We thank the E.C. under project FP6 NMP4-CT-2003505699 for support.
*Present address: IPCMS/GONLO, 23, rue du Loess, 67034 Strasbourg Cedex, France.

†resent address: Laboratoire des Matériaux Polyméres et Biomatériaux, Université Claude Bernard Lyon I, 43, boulevard du 11 novembre 1918, 69622 Villeurbanne Cedex, France.

¥Author to whom correspondence should be addressed. Email address: ebbesen@isis-ulp.org
${ }^{1}$ W. L. Barnes, A. Dereux, and T. W. Ebbesen, Nature (London) 424, 824 (2003).

${ }^{2}$ T. W. Ebbesen, H. J. Lezec, H. F. Ghaemi, T. Thio, and P. A. Wolff, Nature (London) 391, 667 (1998).

${ }^{3}$ H. F. Ghaemi, T. Thio, D. E. Grupp, and H. J. Lezec, Phys. Rev. B 58, 6779 (1998).

${ }^{4}$ L. Martin-Moreno, F. J. Garcia-Vidal, H. J. Lezec, K. M. Pellerin, 
T. Thio, J. B. Pendry, and T. W. Ebbesen, Phys. Rev. Lett. 86, 1114 (2001).

${ }^{5}$ A. Degiron, H. J. Lezec, W. L. Barnes, and T. W. Ebbesen, Appl. Phys. Lett. 81, 4327 (2002).

${ }^{6}$ W. L. Barnes, W. A. Murray, J. Dintinger, E. Devaux, and T. W. Ebbesen, Phys. Rev. Lett. 92, 107401 (2004).

${ }^{7}$ S. M. Williams, A. D. Stafford, K. R. Rodriguez, T. M. Rogers, and J. V. Coe, J. Phys. Chem. B 107, 11871 (2003).

${ }^{8}$ S. M. Williams, K. R. Rodriguez, S. Teeters-Kennedy, A. D. Stafford, S. R. Bishop, U. K. Lincoln, and J. V. Coe, J. Phys. Chem. B 108, 11833 (2004).

${ }^{9}$ Y. Liu and S. Blair, Opt. Lett. 28, 507 (2003).

${ }^{10}$ A. G. Brolo, R. Gordon, B. Leathem, and K. L. Kavanagh, Langmuir 20, 4813 (2004).

${ }^{11}$ K. H. Drexhage, Prog. Opt. 12, 165 (1974).

${ }^{12}$ W. L. Barnes, J. Mod. Opt. 45, 661 (1998).

${ }^{13}$ P. Andrew and W. L. Barnes, Phys. Rev. B 64, 125405 (2001).

${ }^{14}$ I. Pockrand, J. D. Swalen, R. Santo, A. Brillante, and M. R. Philpott, J. Chem. Phys. 69, 4001 (1978).

${ }^{15}$ I. Pockrand and A. Brillante, J. Chem. Phys. 77, 6289 (1982).

${ }^{16}$ M. Brune, F. Schmidt-Kaler, A. Maali, J. Dreyer, E. Hagley, J. M. Raimond, and S. Haroche, Phys. Rev. Lett. 76, 1800 (1996).

${ }^{17}$ C. Weisbuch, M. Nishioka, A. Ishikawa, and Y. Arakawa, Phys. Rev. Lett. 69, 3314 (1992).

${ }^{18}$ M. S. Skolnick, T. A. Fisher, and D. M. Whittaker, Semicond. Sci. Technol. 13, 645 (1998).

${ }^{19}$ A. Christ, S. G. Tikhodeev, N. A. Gippius, J. Kuhl, and H. Giessen, Phys. Rev. Lett. 91, 183901 (2003).
${ }^{20}$ T. Fujita, Y. Sato, T. Kuitani, and T. Ishihara, Phys. Rev. B 57, 12428 (1998).

${ }^{21}$ D. G. Lidzey, D. D. C. Bradley, M. S. Skolnick, T. Virgili, S. Walker, and D. M. Whittaker, Nature (London) 395, 53 (1998).

${ }^{22}$ D. G. Lidzey, D. D. C. Bradley, A. Armitage, S. Walker, and M. S. Skolnick, Science 288, 1620 (2000).

${ }^{23}$ P. A. Hobson, W. L. Barnes, D. G. Lidzey, G. A. Gehring, D. M. Whittaker, M. S. Skolnick, and S. Walker, Appl. Phys. Lett. 81, 3519 (2002)

${ }^{24}$ D. M. Whittaker, Phys. Rev. Lett. 80, 4791 (1998).

${ }^{25}$ C. Ell, J. Prineas, T. R. Nelson, S. Park, H. M. Gibbs, G. Khitorova, S. W. Koch, and R. Houdré, Phys. Rev. Lett. 80, 4795 (1998).

${ }^{26}$ A. Dogariu, T. Thio, L. J. Wang, T. W. Ebbesen, and H. J. Lezec, Opt. Lett. 26, 450 (2001).

${ }^{27}$ D. S. Kim, S. C. Hohng, V. Malyarchuk, Y. C. Yoon, Y. H. Ahn, K. J. Yee, J. W. Park, J. Kim, Q. H. Park, and C. Lienau, Phys. Rev. Lett. 91, 143901 (2003).

${ }^{28}$ D. L. Akins, in J-Aggregates, edited by T. Kobayashi (World Scientific, Singapore, 1996), Chap. 2.

${ }^{29}$ D. W. Lynch and W. R. Hunter, in Handbook of Optical Constants of Solids, edited by E. P. Palik (Academic, Orlando, 1985).

${ }^{30}$ Since submission of this manuscript, an article on strong coupling involving surface plasmons has appeared: see J. Bellessa, C. Bonnand, J. C. Plenet, and J. Mugnier, Phys. Rev. Lett. 93, 036404 (2004). 